\title{
Actualidad extranjera
}

\author{
ANÍBAL ZÁRATE ${ }^{1}$ \\ JuAn Carlos Covilla ${ }^{2}$
}

La Autoridad de la Competencia francesa (AFC) en opinión n. ${ }^{\circ}$ 16-A-05 del 16 de febrero de 2016 se pronunció sobre la conformidad con las normas de competencia de las reglas de la contratación pública relativas a las ofertas que no satisfacen exigencias y especificaciones contenidas en los pliegos de condiciones.

La opinión de la Autoridad se da en respuesta a la consulta elevada por la comuna de Saint-Germain-en-Laye ${ }^{3}$, la cual manifiesta que las normas de la contratación pública contienen disposiciones excesivamente rígidas, en especial las de los procesos de licitaciones públicas y de la regularización de aquellas ofertas llamadas "no conformes" ${ }^{4}$, situación que ha llevado a la comuna en di-

1 Doctor en Derecho de la Universidad Panthéon-Assas, Paris II, Paris, Francia. Docente investigador y director del Grupo de Investigación del Departamento de Derecho Administrativo de la Universidad Externado de Colombia, Bogotá, Colombia. Correo-e: anibal. zarate@uexternado.edu.co Para citar el artículo: Zárate, A. y Covilla, J. C. (2016). Actualidad Extranjera. Revista digital de Derecho Administrativo n. ${ }^{\circ} 15$, primer semestre, Universidad Externado de Colombia, pp. 11-17, DOI: http://dx.doi.org/10.18601/21452946.n15.03.

2 Abogado y magíster en Derecho Administrativo de la Universidad Externado de Colombia Bogotá, Colombia. Candidato a Doctor en Derecho y Ciencia Política de la Universidad de Barcelona, Barcelona, España. Docente e investigador del Departamento de Derecho Administrativo de la Universidad Externado de Colombia, Bogotá, Colombia. Correo-e: juan.covilla@uexternado.edu.co

3 Mediante solicitud radicada el 8 de septiembre de 2015, con el número 15/0076A, y carta de 15 de octubre de 2015.

4 En derecho francés, las ofertas no conformes corresponden a aquellas irregulares, inaceptables e inapropiadas: "Les offres dites 'non conformes' correspondent, en droit, aux offres irrégulières, inacceptables et inappropriés" (AFC, Avis n. ${ }^{\circ}$ 16-A-05, punto 27). La Directiva n. ${ }^{\circ}$ 2004/18/CE de 31 de marzo de 2004, relativa a la coordinación de procesos de selección para la celebración de contratos de obra, suministros y servicios, aplicable hasta abril de este año, se refiere a las nociones de ofertas irregulares y ofertas inaceptables pero no las define. En el derecho francés, el artículo 35 del Código de la Contratos Públicos (Code des marchés publics) define la oferta no conforme como aquella que puede ser: 1. Una oferta irregular, esto es, aquella que "a pesar de aportar una respuesta al poder adjudicador, está incompleta o no respeta las exigencias formuladas en los pliegos de condiciones o en los documentos del proceso"; 2. Una oferta inaceptable, la cual se presenta si las condiciones que están previstas para su ejecución desconocen la legislación vigente o si los créditos presupuestales asignados al contrato luego de la evaluación de la necesidad que se busca satisfacer no permiten al poder adjudicador financiarla ${ }_{i}$ o bien, 3 . Una oferta inapropiada, la cual 
ferentes ocasiones a descartar ofertas presentadas por diferentes proponentes, siendo que esas irregularidades eran mínimas ${ }^{5}$.

A pesar de que la incidencia de esta problemática sobre las cuestiones propias del derecho de la competencia no es evidente ${ }^{6}$, la Autoridad considera que la solicitud de opinión de la comuna de Saint-Germain-en Laye es admisible. La solicitud de la comuna se inscribe además en el contexto particular de la reforma de las normas que integran el estatuto de la contratación pública que el ministro de Economía anunció sería presentada a mediados de 2016, y en la perspectiva de la adopción de un proyecto de decreto relativo a la contratación pública, que en su artículo $61^{[7]}$ autoriza a las entidades contratantes a invitar a los proponentes a corregir las ofertas "no conformes", en el marco de los procesos de licitaciones públicas en curso. En ejercicio de una función similar a la de abogacía de la competencia, que en derecho colombiano se encuentra prevista en el artículo $7 .^{\circ}$ de la Ley 1340 de 2009, la AFC decide participar en el debate en torno a la evolución del derecho de la contratación pública, pese a no estar formalmente invitada por los autores de estas reformas ${ }^{8}$. En este orden de ideas, ella aprovecha la ocasión para expresar su deseo de ser consultada de

se configura cuando aporta una respuesta que no guarda relación con la necesidad de la entidad pública y que en consecuencia puede ser considerada como una ausencia de oferta.

En concreto, "elle soutient que ces modalités l'ont conduite à écarter des offres présentées par différents candidats comme 'non conformes' alors que les irrégularités en cause étaient minimes".

6 AFC. Avis n. ${ }^{\circ} 00-\mathrm{A}-25$ y Avis n. ${ }^{\circ} 03-\mathrm{A}-05$.

7 La norma busca unificar las reglas para la regularización de las ofertas "no conformes". Se trataría solo de aquellas irregulares (ver supra nota 2). El texto de la reforma permite a los proponentes modificar sus ofertas irregulares, sin que esto comporte una modificación de las características esenciales de las mismas. Pero, ¿cuáles son estas características esenciales? Sobre este punto la opinión de la AFC no se pronuncia. En Colombia existe la posibilidad de hacer realizar esas "correcciones", subsanando así las propuestas presentadas. Un avance considerable en la materia se dio mediante un cambio reciente en la jurisprudencia del Consejo de Estado. En el fallo respectivo se indicó que los proponentes tienen la posibilidad de subsanar todos aquellos defectos presentados en la prueba del cumplimiento de los requisitos habilitantes de la propuesta, esto es, aquellos que se refieren a la persona que presenta la oferta y no a la oferta misma. Ver Consejo de Estado, Sala de lo Contencioso Administrativo, Sección Tercera, Subsección A, Sentencia de 12 de noviembre de 2014, exp. 29.855

8 Conforme a lo dispuesto en el artículo 7. ${ }^{\circ}$ de la Ley 1340 de 2009, siendo la Superintendencia de Industria y Comercio la autoridad única de competencia en Colombia, "Además de las disposiciones consagradas en el artículo 2. ${ }^{\circ}$ del Decreto 2153 de 1992, la Superintendencia de Industria y Comercio podrá rendir concepto previo sobre los proyectos de regulación estatal que puedan tener incidencia sobre la libre competencia en los mercados. Para estos efectos, las autoridades de regulación informarán a la Superintendencia de Industria y Comercio de los actos administrativos que se pretenda expedir. El concepto emitido por la Superintendencia de Industria y Comercio en este sentido no será vinculante. Sin embargo, si la autoridad respectiva se apartara de dicho concepto, la misma deberá manifestar de manera expresa dentro de las consideraciones de la decisión los motivos por los cuales se aparta". 
ahora en adelante cada vez que textos legislativos o de carácter reglamentario que modifiquen las normas de la contratación pública se encuentren en preparación, y por las administraciones nacionales implicadas en la negociación de los textos europeos relativos al sistema de compras públicas ${ }^{9}$.

La AFC presentó sus recomendaciones luego de que sus servicios de instrucción interrogaran tanto a los jefes o representantes de entidades contratantes como de empresas contratistas, en particular sobre estos tres puntos: 1. ¿Hasta dónde conviene flexibilizar los dispositivos previstos para ofertas "no conformes"?; 2. ¿Es necesario informar a otros oferentes? Y, en caso de aquí sea, ¿qué informaciones deben comunicarse?; 3 . ¿Conviene imponer a las entidades contratantes el deber de solicitar a los oferentes que regularicen sus ofertas, en especial en el caso en que una sola de las ofertas presentadas sea regular?

Con relación a la primera pregunta, la AFC se muestra favorable a una flexibilización, aunque limitada, de las normas que rigen las ofertas "no conformes". Considera que una flexibilización del dispositivo existente para el trato de ofertas "no conformes" y una mayor discrecionalidad conferida a la entidad contratante para reglar este asunto serían favorables a la libre competencia económica. Esto, por cuanto permite abandonar un sistema que lleva a descartar de forma casi mecánica ciertas ofertas, por lo que un mayor número de oferentes podrían seguir compitiendo por la adjudicación del contrato ${ }^{10}$. No obstante, la AFC estima que una flexibilización excesiva de las normas de la contratación pública sobre este punto podría traducirse en una especie "negociación" de las ofertas y favorecer la aparición de situaciones discriminatorias contrarias a la competencia ${ }^{11}$. En efecto, admitir que la regularización de las ofertas irregulares puede llevar a modificar las ofertas en sus características "no esenciales" comporta riesgos como la colusión en licitaciones públicas. La AFC estima por consiguiente que la regularización debe estar limitada a la corrección de errores y omisiones formales, que en sí mismos no tienen una incidencia sobre las

9 Es preciso recordar que la reforma francesa se inscribe en el marco de la adopción de tres directivas que revisan las normas de la contratación pública y en materia de concesiones: 1. La Directiva n. ${ }^{\circ}$ 2014/23/ue del Parlamento y del Consejo europeos de 26 de febrero de 2014, relativa a la adjudicación de los contratos de concesión (JO L 94, 28.2.2014, pp. 1-64); 2. La Directiva n. ${ }^{\circ}$ 2014/24/UE del Parlamento y del Consejo europeos sobre la celebración de contratos públicos (JO L 94, 28.3.2014, pp. 65-242); y, 3. La Directiva n. ${ }^{\circ}$ 2014/25/Ue del Parlamento y del Consejo europeos de 26 de febrero de 2014, sobre la celebración de contratos por las entidades que operan en los sectores de energía, agua, transportes y servicios postales (JO L 94, 28.3.2014, pp. 243-374).

10 AFC. Avis n. ${ }^{\circ}$ 16-A-05, punto 133.

11 Para la AFC es imperativo que las reglas de la contratación pública no faciliten o incentiven prácticas anticompetitivas como los acuerdos o el abuso de posición dominante. "Cet aspect est d'autant plus important que les règles actuelles de l'achat public coexistent avec des pratiques d'entente entre les entreprises qui répondent aux appels d'offres et qui aboutissent souvent à une bausse significative du prix payé par l'acheteur public, en contradiction avec l'objectif recherché par le droit de la commande publique" (AFC. Avis n. ${ }^{\circ}$ 00-A-25 y Avis n. ${ }^{\circ} 03-\mathrm{A}-05$, puntos 4 a 6 ). 
características técnicas y económicas de las ofertas ${ }^{12}$. Comparativamente, en Colombia los proponentes pueden subsanar ciertos requisitos habilitantes ${ }^{13}$, pero sin que esto comporte la modificación de lo que la jurisprudencia del Consejo de Estado ha denominado "aquello que otorga puntaje" y que realmente consiste en la parte sustancial de la propuesta presentada ${ }^{14}$.

Por el contrario, en el caso en que los autores de la reforma decidan abrir la vía a la regularización de ofertas irregulares, con la condición de que no sea

12 AFC. Avis n. ${ }^{\circ}$ 16-A-05, puntos 134 y 135. La posición de la Autoridad parece mantenerse al margen del debate sobre qué se entiende como sustancial de la oferta. En Colombia, la jurisprudencia del Consejo de Estado establece que no se puede subsanar lo que otorga puntaje, esto es, la oferta técnica y la oferta económica: ver, por ejemplo, Consejo de Estado, Sección Tercera, 26 de febrero de 2014, exp. 25804: "En adelante, el criterio de diferenciación entre los requisitos subsanables y no subsanables de una oferta incompleta dejó de ser, en abstracto, 'aquello que sea o no necesario para la comparación de las ofertas' y pasó a ser todavía parte de eso, pero bajo un entendimiento más concreto, menos abstracto o indeterminado: ahora son subsanables '... todos aquellos requisitos de la propuesta que no afecten la asignación de puntaje'. Es además preciso diferenciar la subsanabilidad de los requisitos habilitantes consagrada en el parágrafo 1 del artículo 5 de la Ley 1150 de 2007 de la posibilidad de aclarar y explicar las ofertas prevista en el artículo 30.7 de la Ley 80 de 1993". En esta misma jurisprudencia del Consejo de Estado de febrero de 2014 se señala que "subsanar no es lo mismo que aclarar o explicar, aunque en ocasiones aquél se use como consecuencia de las explicaciones dadas. La importancia de diferenciarlos radica en que la aclaración o explicación se admite, incluso, sobre requisitos que afectan la comparación de las ofertas y/o inciden en la asignación de puntaje".

13 Arts. 5. ${ }^{\circ}$ Ley 1150 de 2007 y 10. ${ }^{\circ}$ Decreto 1510 de 2013: "Los requisitos habilitantes miden la aptitud del proponente para participar en un Proceso de contratación como oferente y están referidos a su capacidad jurídica, financiera, organizacional y su experiencia". Conforme al manual para determinar y verificar los requisitos habilitantes en los procesos de contratación de la Agencia nacional "Colombia compra eficiente", "El propósito de los requisitos habilitantes es establecer unas condiciones mínimas para los proponentes de tal manera que la entidad estatal solo evalúe las ofertas de aquellos que están en condiciones de cumplir con el objeto del proceso de contratación".

14 Consejo de Estado, Sección Tercera. Sentencia de 12 de noviembre de 2014, exp. 27896. Sobre este asunto, en Colombia se tiene un avance considerable en la medida que se pueden identificar claramente los casos en que una parte de la propuesta puede ser subsanada y los casos en que no, teniendo en cuenta la diferenciación de los aspectos que se refieren al proponente y los que se refieren a la oferta presentada. Los primeros se pueden subsanar en cualquier momento hasta la adjudicación y los segundos no se pueden subsanar (ver Consejo de Estado, Sección Tercera. Sentencia de 26 de febrero de 2014, exp. 25804: "podrán ser solicitados por las entidades en cualquier momento, hasta la adjudicación"). No es propiamente una medida flexible, pues la entidad contratante solamente revisa el tipo de requisito que está incorrecto y decide si se trata de los que pueden ser subsanados o no, pero por lo menos tiene el oferente la seguridad de que los subsanables son aquellos que no modifican la oferta que presenta. A su vez, cuando se trate de un requisito no subsanable, bien puede aclarar alguno de sus aspectos, si es oscuro para la entidad contratante y así lo solicita, lo que no implica corregir la propuesta, sino eliminar aspectos poco claros para el evaluador. Ver Consejo de Estado, Sala de lo Contencioso Administrativo, Sección Tercera, Subsección C, Sentencia de 26 de noviembre de 2015, exp. 51376. 
posible modificar sus características sustanciales, la AFC recomienda recordar el carácter intangible de la oferta y precisar que en ningún caso esta modificación puede comportar una modificación de las condiciones y especificaciones técnicas y económicas que se encuentran en los pliegos de condiciones.

Además, la AFC estima que será necesario dar ejemplos concretos de aque1lo que constituye "las características sustanciales" de una oferta y de aquello que no lo es a las entidades contratantes, quienes, como la comuna de SaintGermain-en-Laye, solicitan se aclare el alcance que tendrá el proyecto de reforma ${ }^{15}$. La Autoridad recomienda en su opinión que, al recibir ofertas "no conformes", la entidad garantice un trato igual a todos los oferentes, por lo que las administraciones contratantes deberán invitar a cada una de las personas naturales o jurídicas oferentes a regularizar su oferta, y no únicamente a algunas de ellas. Y señala además que se debe fijar el término o plazo durante el cual las ofertas podrán ser regularizadas, indicando por ejemplo que esta fase no podrá exceder de 10 días ${ }^{16}$.

Tratándose de la segunda pregunta, esto es, la referida a la información de los otros oferentes, la AFC considera que la obligación de informar a los candidatos responde al objetivo legítimo de preservar la igualdad entre los mismos y que la transparencia que ello aporta es indispensable para el equilibrio del dispositivo de regularización contenido en el proyecto de decreto. En efecto, la información a los oferentes invitados a regularizar sus ofertas debe permitirles interponer un recurso cuando se estimen vulnerados en sus derechos, así como denunciar eventuales discriminaciones. Ahora bien, la información dada a los candidatos podría ser susceptible de facilitar prácticas contrarias a la libre competencia económica entre los oferentes y, en particular, ciertos intercambios entre las empresas oferentes. De ahí que la AFC estime que esta información deba estar estrictamente encuadrada y delimitada ${ }^{17}$. La Autoridad recomienda por tanto que cuando la entidad contratante autorice la regularización de ofertas a los proponentes que participan en un proceso de licitación, esta informe a los otros oferentes el plazo que tienen para interponer los recursos a que haya lugar. Pero recomienda asimismo que esta información no revele ni la identidad de las empresas oferentes involucradas ni el contenido de sus ofertas, sino únicamente la naturaleza o el tipo de regularización que se permite para ese proceso de selección ${ }^{18}$. La situación en Colombia es diferente. En aras de la transparencia y publicidad, la solicitud de aclaraciones a las propuestas previo al informe de evaluación y el informe de evaluación mismo trata de ser lo más detallada posible en cuanto a la solicitud de correcciones, contratación pública. AFC. Avis n. ${ }^{\circ}$ 16-A-05, punto 136. 
lo que ha llevado a que los demás oferentes se pronuncien sobre la posibilidad de regularizarla. Incluso, en la etapa misma de traslado del informe de evaluación según el artículo 30.8 de la Ley 80 de 1993, se permite que los oferentes revisen las propuestas del resto para poder constatar si estas contienen errores que pueden o no ser subsanados. En estos casos, los oferentes parecen jugar un rol de verificadores de la evaluación.

Ahora bien, conforme a las recomendaciones formuladas en 2012 por la Organización para la Cooperación y el Desarrollo Económico-OCDE- ${ }^{19}$, el diseño de los procesos de adquisición pública debe buscar reducir los riesgos de colusión en la presentación de ofertas ${ }^{20}$. En este orden de ideas, si bien reconoce que la transparencia es vital para los procesos de adquisición pública, señala que esta puede llegar a ser problemática cuando se aplica indiscriminadamente a la difusión de información sensible. En efecto, los lineamientos de la OCDE destacan el potencial para la colusión durante una licitación cuando los oferentes disponen de los medios para conocer la identidad de sus competidores en potencia, facilitando que se reúnan con ellos ${ }^{21}$. De ahí que aconseje que los oferentes sean identificados por letras o por números, no por sus nombres. Además, dentro de las informaciones que se aconseja a las autoridades colombianas que eviten divulgar a los oferentes se incluyen la oferta económica, las minutas de las reuniones de aclaración, y la información sobre contratos adjudicados y cumplidos por proveedores individuales. Como alternativa, la OCDE sugiere que se podría poner a disposición información más completa con cierto lapso de tiempo intermedio, esto es, de "más de seis meses después

19 Organización para la Cooperación y el Desarrollo Económico-OCDE-. Recomendación del Consejo de la OCDE para combatir la colusión en la contratación pública, 2012, disponible en: http:// www.oecd.org/daf/competition/Recommendation_fighting-bid-rigging-2012-ES.pdf [consultado: 10 de mayo de 2016].

20 En particular encuentra que es necesario diseñar el proceso de licitación para reducir eficazmente la comunicación entre oferentes: ver OCDE. Ob. cit., p. 12.

21 "La experiencia internacional confirma que las reuniones aclaratorias con los oferentes ordenadas por la legislación de adquisiciones públicas, como es el caso de Colombia, facilitan un foro en el que los oferentes pueden discutir o culminar un acuerdo, o intercambiar información sensible en detrimento de la competencia. Además, las visitas sobre el terreno, las listas de los que han solicitado información sobre los oferentes o expresado interés en la licitación, las listas de oferentes, las aperturas públicas de las ofertas y la divulgación del precio de la proposición presentada por cada oferente, han sido identificados en los Lineamientos de la OCDE como acciones que hay que evitar en licitaciones. Cuando no sea posible eliminar la oportunidad de que los oferentes potenciales se conozcan e interactúen, esas prácticas deberían ser minimizadas y vigiladas con atención": Organización para la Cooperación y el Desarrollo Económico-OCDE-. Combatiendo la colusión en los procesos de contratación pública en Colombia. Informe del Secretariado sobre el marco jurídico y las prácticas de la contratación pública en Colombia, 2014, p. 49. Disponible en: https://www.oecd.org/daf/ competition/2014_Fighting\%20Bid\%20Rigging\%20Colombia_SPA.pdf, [consultado: 10 de mayo de 2016]. 
de la contratación pública". Ello con el fin de obstaculizar o alterar el control y el cumplimiento de un plan colusorio de los participantes ${ }^{22}$.

En relación con la tercera y última pregunta, la Autoridad se muestra hostil a la instauración de una obligación para la entidad contratante de solicitar la regularización de las ofertas irregulares. Ella considera que, en el contexto general de una reforma que busca dar una mayor capacidad de negociación a la Administración pública, una medida en este sentido, consistente en una obligación de solicitar de manera automática la regularización de las ofertas "no conformes" irregulares, podría ser contraria a esta intención. Al mismo tiempo, ella no encuentra una justificación a la luz de las reglas de la libre competencia económica y, en este sentido, se considera que no es necesaria para favorecer la competencia entre oferentes en los procesos licitatorios. Por ende, la Autoridad recomienda que las entidades públicas dispongan de un margen de discrecionalidad que les permita poner o no en obra un procedimiento de regularización de las ofertas irregulares, más que imponerles una obligación, incluso cuando solo una de las ofertas es regular ${ }^{23}$.

Si bien la situación del derecho francés en comparación con el derecho colombiano en materia de regularización de las ofertas presentadas es diferente, esta opinión de la AFC sirve también para reflexionar en Colombia sobre la necesidad de que la contratación pública no sólo se estudie a partir de la sustantividad que tiene como parte del derecho administrativo, sino que también se revise a la luz del derecho de la competencia, pues aquí también juega un papel importante. En esto ha sido fundamental la Superintendencia de Industria y Comercio que ha emitido conceptos y guías desde esta perspectiva ${ }^{24}$.

Es posible consultar la opinión de la AFC en el siguiente vínculo:

http://www.autoritedelaconcurrence.fr/pdf/avis/16a05.pdf

24 Al respecto ver Superintendencia de Industria y Comercio y Ministerio de Industria y Comercio. Guía Práctica para Combatir la Colusión en las Licitaciones, disponible en: http://www.sic.gov.co/recursos_user/documentos/articulos/2010/Guia_Contratacion.pdf 